\title{
AVAILABILITY OF FISH FAUNA IN SOME SELECTED DISTRICTS IN BANGLADESH
}

\author{
K. J. Chandra ${ }^{1}$
}

\begin{abstract}
A check-list of 139 species of fishes of both exotic and indigenous origin belonging to 34 families and 87 genera available in the rivers, beels, haors, ponds and swamps of the greater Mymensingh (Mymensingh, Kishoregonj, Netrakona, Jamalpur and Sherpur) districts of Bangladesh is presented. The number of species, genera and family of fishes reported in Mymensingh regions is still rich in fresh water fish fauna. The systematic, ecology, Distribution, habits of these fish fauna of greater Mymensingh region need to be studied.
\end{abstract}

Key words: Freshwater, Fish fauna, Bangladesh

\section{Introduction}

The first scientific account of freshwater fish fauna of Bangladesh began with the work of Hamilton (1822). Recently, Rahman (2005) has made significant contribution on freshwater fishes and recorded 265 species of fish under 55 families from Bangladesh. A number of other workers described freshwater fish fauna from different parts of the country. Bhuiyan (1964) described 71 species of fish from Dhaka district. Ahmed and Hasan (1981) made a check-list of the fishes of Karnaphuli reservoir. Chandra and Aminul Haque (1982a, 1982b) studies the distribution, abundance, fishery, and taxonomic status of Mohashol (Tor spp.) and identified two species, Tor tor and Tor putitora are distributed in the hill streams of Bangladesh. Islam and Hossain (1983) made an account of 110 species of fish from the river Padma. Haque (2004) recorded 134 species of fish from the capture fisheries of Padma. Hossain et al. (2007) recorded 97 species of teleost from the river Naaf of Chittagong.

The greater Mymensingh (Mymensingh, Kishoregonj, Netrakona, Jamalpur, and Sherpur districts) is rich in freshwater fishes. Mymensingh town is situated on the bank of the river Brahmaputra. Kishoregonj and Netrakona districts are important for haors and beels and these are the prime important for open water fisheries resources. Only a few years back, huge number of fish species were found in Mymensingh which are drastically declining day by day because of various reasons. However, study of fish fauna, Mymensingh in particular is lacking except that of Doha (1973) who reported 106 species of fish from the waters of Mymensingh and Tangail districts. The present report is therefore, an attempt to make a fish faunal list of available species of greater Mymensingh.

Department of Aquaculture, Bangladesh Agricultural University, Mymensingh-2202, Bangladesh

(Received: August 24, 2009) 
Bang. J. Anim. Sci. 2009, 38(1\&2)

\section{Materials and Methods}

The account has been made mainly on the basis of recent reports of different workers on fish fauna and fish biodiversity of different rivers, beels, haors, ponds and swamps of greater Mymensingh region (Rahman, 2000; Wahab, 2003; Chakraborty and Mirza, 2006; Faroque, 2006; Hossain, 2007; Alam, 2008; Dowla, 2008; Rahman, 2009; Debnath, 2009; and Akter, 2009). These fishes were collected with seine nets, dip nets, cast nets, gill nets, traps, hooks and wounding gears. Personal visits to different fishing and landing centres and fish markets were made and many samples collected and preserved in 10 per cent formalin. In several occasions consultation with the fishermen, fish traders and fish farmers were arranged in the fish markets and fish landing points of Mymensingh during 2007-2009. Identification and systematic account of these fishes have presented following Talwar and Jhingran (1991), Rahman (2005) and Siddiqui et al. (2007) with local and English names and the level of national status of availability (IUCN, 2000).

\section{Results and Discussions}

One hundred and thirty nine species of freshwater fishes (126 indigenous and 13 exotic species) belonging 34 families and 87 genera has been reported in this work from greater Mymensingh (Table 1).

Table 1. A check-list of 139 species of fish fauna of greater Mymensingh, Bangladesh

\begin{tabular}{|c|c|c|c|}
\hline Specific names with Taxonomic position & English name & Local name & $\begin{array}{c}\text { Status of } \\
\text { availability }\end{array}$ \\
\hline \multicolumn{4}{|l|}{ Order: OSTEOGLOSSIFORMES } \\
\hline \multicolumn{4}{|l|}{ I. Family: NOTOPTERIDAE } \\
\hline \multicolumn{4}{|l|}{ Genus: Notopterus Lacepede,1800 } \\
\hline 1. Notopterus chitala (Hamilton , 1822) & Humped featherback & Chital & EN \\
\hline 2. Notopterus notopterus (Pallas, 1769) & Gray featherback & Foli, Kanla & Vu \\
\hline \multicolumn{4}{|l|}{ Order: ANGUILLIFORMES } \\
\hline \multicolumn{4}{|l|}{ II. Family: ANGUILIDAE } \\
\hline \multicolumn{4}{|l|}{ Genus: Anguilla Schrank, 1798} \\
\hline 3. Anguilla bengalensis (Gray, 1831) & Longfin Eel & Bamosh & VU \\
\hline \multicolumn{4}{|l|}{ Order: CLUPEIFORMES } \\
\hline \multicolumn{4}{|l|}{ III. Family: CLUPEIDAE } \\
\hline Genus: Corica Hamilton, 1822 & & & \\
\hline
\end{tabular}

Table 1. Continue 
Table 1. Continued

\begin{tabular}{|c|c|c|c|}
\hline Specific names with Taxonomic position & English name & Local name & $\begin{array}{c}\text { Status of } \\
\text { availability }\end{array}$ \\
\hline $\begin{array}{l}\text { 4. Corica soborna Hamilton, } 1822 \\
\text { Genus: Gonialosa Regan, } 1917\end{array}$ & Ganges River-sprat & Kachki & NT \\
\hline $\begin{array}{l}\text { 5. Gonialosa manmina (Hamilton, 1822) } \\
\text { Genus: Tenualosa Fowler, } 1934\end{array}$ & Ganges River Shad & Chapila & NT \\
\hline 6. Tenualosa ilisha (Hamilton, 1822) & River Shad & Ilish, Ilsha & NT \\
\hline $\begin{array}{l}7 \text { Tenualosa toli (Valenciennes, 1847) } \\
\text { IV. Family: ENGRAULIDAE } \\
\text { Genus: Gudusia Fowler, } 1911\end{array}$ & Toli Shad & Chandana Ilish & NT \\
\hline $\begin{array}{l}\text { 8. Gudusia chapra (Hamilton, 1822) } \\
\text { Genus: Setipinna Swainson, } 1839\end{array}$ & Indian River Shad & Chapila, Chapla & DD \\
\hline $\begin{array}{l}\text { 9. Setipinna phasa (Hamilton, 1822) } \\
\text { Order: CHANNIFORMES } \\
\text { V. Family: CHANNIDAE } \\
\text { Genus: Channa Scopoli, } 1777\end{array}$ & Hairfin Anchovy & Phasa & NT \\
\hline 10. Channa punctatus (Bloch, 1793) & Spotted snakehead & Taki, Lata & NT \\
\hline 11. Channa striatus (Bloch, 1793) & Stripped snakehead & Shol & NT \\
\hline 12. Channa orientalis (Bloch \&Schneider, 1801) & Walking snakehead & Cheng, Gachua & EN \\
\hline 13. Channa marulius (Hamilton, 1822) & Giant snakehead & Gajar & EN \\
\hline $\begin{array}{l}\text { 14. Channa barca (Hamilton, 1822) } \\
\text { Order: CYPRINIFORMES } \\
\text { VI. Family: CYPRINIDAE } \\
\text { Genus: Amblypharyngodon Bleeker, } 1860\end{array}$ & Barca snakehead & Til shol, Pipla & $\mathrm{CR}$ \\
\hline $\begin{array}{l}\text { 15. Amblypharyngodon microlepis (Bleeker, } \\
\text { 1853) }\end{array}$ & Indian Carplet & Mola & NT \\
\hline $\begin{array}{l}\text { 16. Amblypharyngodon mola (Hamilton, 1822) } \\
\text { Genus: Aristichthys Oshima, } 1919\end{array}$ & Mola Carplet & Mola, Moa & NT \\
\hline $\begin{array}{l}\text { 17. Aristichthys nobilis (Richardson, 1844)* } \\
\text { Genus: Aspidoparia Heckel, } 1843\end{array}$ & Bighead & Bighead & NL \\
\hline $\begin{array}{l}\text { 18. Aspidoparia jaya (Hamilton, 1822) } \\
\text { Genus: Barbonymus Kottelat, } 1999\end{array}$ & Jaya & Jaya & NT \\
\hline
\end{tabular}

Table 1. Continue 
Bang. J. Anim. Sci. 2009, 38(1\&2)

Table 1. Continued

\begin{tabular}{|c|c|c|c|}
\hline Specific names with Taxonomic position & English name & Local name & $\begin{array}{c}\text { Status of } \\
\text { availability }\end{array}$ \\
\hline 19. Barbonymus gonionotus (Bleeker, 1850)* & Java Barb & Thai Sarputi & $\mathrm{NL}$ \\
\hline \multicolumn{4}{|l|}{ Genus: Barilius Hamilton, 1822} \\
\hline 20. Barilius barna (Hamilton, 1822) & Baril & Koksa & DD \\
\hline 21. Barilius bendelisis (Hamilton, 1807) & Barila & Loia, Koksa & EN \\
\hline 22. Barilius shacra (Hamilton, 1822) & Shacra Baril & Koksa & DD \\
\hline 23. Barilius tileo (Hamilton, 1822) & Tileo Baril & Tila Koksa & DD \\
\hline \multicolumn{4}{|l|}{ Genus: Bengala Gray, 1833} \\
\hline 24. Bengala elanga (Hamilton, 1822) & Bengala Barb & Elong & EN \\
\hline Genus: Catla Valencinnes, 1844 & & & \\
\hline 25. Catla catla (Hamilton, 1822) & Catla & Catla, Katal & NT \\
\hline \multicolumn{4}{|l|}{ Genus: Chagunius Smith, 1938} \\
\hline 26. Chagunius chagunio (Hamilton, 1822) & Chaguni & Jarua & DD \\
\hline \multicolumn{4}{|l|}{ Genus: Chela Hamilton, 1822} \\
\hline 27. Chela cachius (Hamilton, 1822) & Chela & Chhap Chela & DD \\
\hline 28. Chela laubuca (Hamilton, 1822) & Grass Barb & Kash Khaira & EN \\
\hline \multicolumn{4}{|l|}{ Genus: Cirrhinus Oken, 1817} \\
\hline 29. Cirrhinus cirrhosus (Bloch, 1795) & Mrigal & Mrigal, Mirka & NL \\
\hline 30. Cirrhinus reba (Hamilton, 1822) & Reba & Bhagna, Bata & VU \\
\hline \multicolumn{4}{|l|}{ Genus: Crossocheilus van Hasselt, 1823} \\
\hline 31. Crossocheilus latius (Hamilton, 1822) & Gangetic Latia & Kala Bata & EN \\
\hline \multicolumn{4}{|l|}{ Genus Ctenopharyngodon Steindachner, 1866} \\
\hline 32. Ctenopharyngodon idella (Valenciennes, 1844)* & Grass Carp & Grass Carp & NL \\
\hline \multicolumn{4}{|l|}{ Genus: Cyprinus Linnaeus, 1758} \\
\hline 33. Cyprinus carpio Linnaeus, $1758^{\star}$ & Common Carp & Carpu & NL \\
\hline \multicolumn{4}{|l|}{ Genus: Danio Hamilton, 1822} \\
\hline 34. Danio dangila (Hamilton, 1822) & Dangila & Nipati & DD \\
\hline 35. Danio rerio (Hamilton, 1822) & Zebra Danio & Nipati & NL \\
\hline \multicolumn{4}{|l|}{ Genus: Devario Heckel, 1843} \\
\hline 36. Devario acquipinnatus (McClelland, 1839) & Bengal Danio & Chebli & NT \\
\hline 37. Devario devario (Hamilton, 1822) & Danio & Baspata, Chebli & NT \\
\hline Genus: Esomus Swainson, 1839 & & & \\
\hline
\end{tabular}

Table 1. Continue 
Table 1. Continued

\begin{tabular}{|c|c|c|c|}
\hline Specific names with Taxonomic position & English name & Local name & $\begin{array}{l}\text { Status of } \\
\text { availability }\end{array}$ \\
\hline 38. Esomus danricus (Hamilton, 1822) & Flying Barb & Darkina & DD \\
\hline \multicolumn{4}{|l|}{ Genus Hypophthalmichthys Bleeker, 1860} \\
\hline 39. Hypophthalmichthys molitrix (Valenciennes, 1844)* & Silver Carp & Silver Carp & NL \\
\hline \multicolumn{4}{|l|}{ Genus: Labeo Cuvier, 1817} \\
\hline 40. Labeo rohita (Hamilton, 1822) & Rahu & Rui, Rohit & NT \\
\hline 41. Labeo angra (Hamilton, 1822) & Angra Labeo & Angrot, Kharsa & NT \\
\hline 42. Labeo bata (Hamilton, 1822) & Bata Labeo & Bata & EN \\
\hline 43. Labeo calbasu (Hamilton, 1822) & Kalbasu & Kalibaus & EN \\
\hline 44. Labeo dero (Hamilton, 1822) & Kalabans & Kursha & EN \\
\hline 45. Labeo nandina (Hamilton, 1822) & Nandi Labeo & Nandina & $\mathrm{CR}$ \\
\hline 46. Labeo gonius (Hamilton, 1822) & Kuria Labeo & Ghanyya & EN \\
\hline 47. Labeo boga (Hamilton, 1822) & Boga Labeo & Bhangan & $\mathrm{CR}$ \\
\hline \multicolumn{4}{|l|}{ Genus: Mylopharyngodon Peters, 1881} \\
\hline 48. Mylopharyngodon peceus (Richadson, 1846)* & Black Carp & Carp & NL \\
\hline \multicolumn{4}{|l|}{ Genus: Osteobrama Heckel, 1842} \\
\hline 49. Osteobrama cotio (Hamilton, 1822) & Cotio & Dhela & EN \\
\hline \multicolumn{4}{|l|}{ Genus: Puntius Hamilton, 1822} \\
\hline 50. Puntius chola (Hamilton, 1822) & Chola Barb & Chalaputi & NT \\
\hline 51. Puntius phutunio (Hamilton, 1822) & Dwarf Barb & Phutani Punti & NT \\
\hline 52. Puntius conchonius (Hamilton, 1822) & Rosy Barb & Kanchan Punti & NT \\
\hline 53. Puntius gelius (Hamilton, 1822) & Golden Barb & Gili Punti & DD \\
\hline 54. Pontius sarana (Hamilton, 1822) & Olive Barb & Sar Punti & $\mathrm{CR}$ \\
\hline 55. Puntius sophore (Hamilton, 1822) & Pool Barb & Jat Punti & NT \\
\hline 56. Puntius terio (Hamilton, 1822) & One Spot Barb & Teri Punti & NT \\
\hline 57. Puntius ticto (Hamilton, 1822) & Ticto Barb & Tit Punti & VU \\
\hline \multicolumn{4}{|l|}{ Genus: Raiamas Jordan, 1918} \\
\hline 58. Raiamas bola (Hamilton,1822) & Indian Trout & Bhol, Bol & EN \\
\hline \multicolumn{4}{|l|}{ Genus: Rasbora Bleeker, 1860} \\
\hline 59. Rasbora daniconius (Hamilton, 1822) & Rasbora & Darkina & NL \\
\hline 60. Rasbora rasbora (Hamilton, 1822) & Gangetic Rasbora & Darkina & EN \\
\hline \multicolumn{4}{|l|}{ Genus: Salmostoma Swainson, 1839} \\
\hline 61. Salmostoma bacaila (Hamilton, 1822) & $\begin{array}{l}\text { Large Razorbelly } \\
\text { Minnow }\end{array}$ & Katari & NT \\
\hline
\end{tabular}

Table 1. Continue 
Bang. J. Anim. Sci. 2009, 38(1\&2)

Table 1. Continued

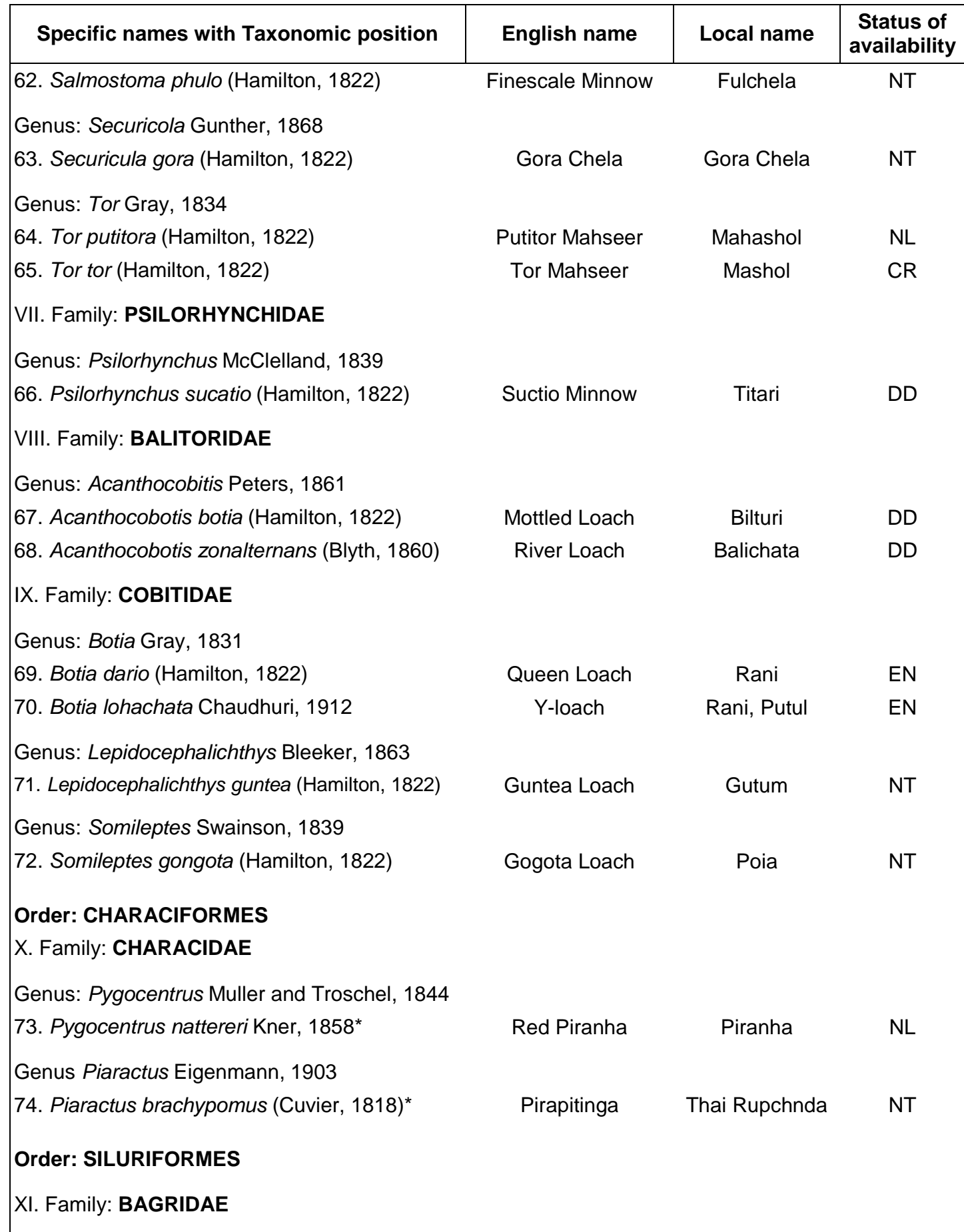

Table 1. Continue 
Table 1. Continued

\begin{tabular}{|c|c|c|c|}
\hline Specific names with Taxonomic position & English name & Local name & $\begin{array}{c}\text { Status of } \\
\text { availability }\end{array}$ \\
\hline \multicolumn{4}{|l|}{ Genus: Batasio Blyth, 1860} \\
\hline 75. Batasio tengana (Hamilton, 1822) & Dwarf catfish & Tengra & EN \\
\hline \multicolumn{4}{|l|}{ Genus: Hemibagrus Bleeker, 1862} \\
\hline 76. Hemibagrus menoda (Hamilton, 1822) & Menoda catfish & Gang Tengra & NT \\
\hline \multicolumn{4}{|l|}{ Genus Mystus Scopoli, 1777} \\
\hline 77. Mystus bleekeri (Day, 1877) & Day's Mystus & Tengra & NT \\
\hline 78. Mystus cavassius (Hamilton, 1822) & Gangetic Mystus & Gulsha & VU \\
\hline 79. Mystus gulio (Hamilton, 1822) & Gulio Catfish & Nuna Tengra & DD \\
\hline 80. Mystus tengara (Hamilton, 1822) & Tengara Mystus & Bajari Tengra & NT \\
\hline 81. Mystus vittatus (Bloch, 1797) & Stripped Dwarf Catfish & Tengra & NT \\
\hline \multicolumn{4}{|l|}{ Genus: Rama Bleeker, 1855} \\
\hline 82. Rama chandramara (Hamilton, 1822) & Asian Cory & Bajaria Tengra & DD \\
\hline \multicolumn{4}{|l|}{ Genus: Rita Bleeker, 1858} \\
\hline 43. Rita rita (Hamilton, 1822) & Rita & Rita & $\mathrm{CR}$ \\
\hline \multicolumn{4}{|l|}{ Genus Sperata Holly, 1939} \\
\hline 84. Sperata aor (Hamilton, 1822) & Lon Wiskered Catfish & Ayre & VU \\
\hline 85. Sperata seenghala (Sykes,1839) & Giant River Catfish & Guijja & EN \\
\hline \multicolumn{4}{|l|}{ XII. Family: SILURIDAE } \\
\hline \multicolumn{4}{|l|}{ Genus: Ompok Lacepede, 1803} \\
\hline 86. Ompok bimaculata (Bloch, 1797) & Butter Catfish & Kani Pabda & EN \\
\hline 87. Ompok pabda (Hamilton, 1822) & Pabda Catfish & Pabda & EN \\
\hline 88. Ompok pabo (Hamilton, 1822) & Pabo Catfish & Kala Pabda & EN \\
\hline \multicolumn{4}{|l|}{ Genus: Wallago Bleeker, 1951} \\
\hline 89. Wallago attu (Schneider, 1801) & Boal & Boal & NT \\
\hline \multicolumn{4}{|l|}{ XIII. Family: SCHILBEIDAE } \\
\hline \multicolumn{4}{|l|}{ Genus Ailia Gray, 1831} \\
\hline 90. Ailia coila (Hamilton, 1822) & Gangetic Ailia & Kajuli & NT \\
\hline \multicolumn{4}{|l|}{ Genus: Clupisoma Swainson, 1839} \\
\hline 91. Clupisoma garua (Hamilton, 1822) & Garua Bacha & Ghaura & $\mathrm{CR}$ \\
\hline \multicolumn{4}{|l|}{ Genus Eutropiichthys Bleeker, 1862} \\
\hline 92. Eutropiichthys murius (Hamilton, 1822) & Murius Bacha & Muri Bacha & NT \\
\hline 93. Eutropiichthys vacha (Hamilton, 1822) & Bacha & Bacha & $\mathrm{CR}$ \\
\hline
\end{tabular}

Table 1. Continue 
Bang. J. Anim. Sci. 2009, 38(1\&2)

Table 1. Continued

\begin{tabular}{|c|c|c|c|}
\hline Specific names with Taxonomic position & English name & Local name & $\begin{array}{l}\text { Status of } \\
\text { availability }\end{array}$ \\
\hline \multicolumn{4}{|l|}{ Genus: Pseudeutropius Bleeker, 1862} \\
\hline 94. Pseudeutropius atherinoides (Bloch, 1794) & Indian Potasi & Batasi & NT \\
\hline \multicolumn{4}{|l|}{ Genus: Silonia Swainson, 1839} \\
\hline 95. Silonia silondia (Hamilton, 1922) & Silond Catfish & Shilong & EN \\
\hline \multicolumn{4}{|l|}{ XIV. PANGASIIDAE } \\
\hline \multicolumn{4}{|l|}{ Genus: Pangusius Valenciennes, 1849} \\
\hline 96. Pangasius pangasius (Hamilton 1822) & Pangus & Pangas & CR \\
\hline 97. Pangasius hypophthalmus (Sauvage, 1878)* & Pungas Catfish & Thai Pangas & NL \\
\hline \multicolumn{4}{|l|}{ XV. Family: AMBLYCIPITIDAE } \\
\hline \multicolumn{4}{|l|}{ Genus: Amblyceps Blyth, 1858} \\
\hline 98. Amblyceps mangois (Hamilton, 1822) & Torrent Catfish & Chhota Shinghi & DD \\
\hline \multicolumn{4}{|l|}{ XVI. Family: SISORIDAE } \\
\hline \multicolumn{4}{|l|}{ Genus: Bagarius Bleeker, 1853} \\
\hline 99. Bagarius bagarius (Hamilton, 1822) & Gangetic Goonch & Baghair & CR \\
\hline \multicolumn{4}{|l|}{ Genus: Gagata Bleeker, 1858} \\
\hline 100. Gagata cenia (Hamilton, 1822) & Indian Gagata & Jungla & NT \\
\hline 101. Gagata gagata (Hamilton, 1822) & Gangetic Gagata & Gang Tengra & NT \\
\hline \multicolumn{4}{|l|}{ Genus: Gogangra Roberts, 2001} \\
\hline 102. Gogangra viridescens (Hamilton, 1822) & Huddah Nangra & Gang Tengra & NT \\
\hline \multicolumn{4}{|l|}{ Genus: Hara Blyth, 1861} \\
\hline 103. Hara hara (Hamilton, 1822) & Kosi Hara & Kutakanti & NT \\
\hline 104. Hara jerdoni Day, 1870 & Sylhet Hara & Kutakanti & NT \\
\hline \multicolumn{4}{|l|}{ Genus: Pseudolaguvia Misra, 1976} \\
\hline 105. Pseudolaguvia inornata Ng, 2005 & Painted Catfish & Kani Tengra & DD \\
\hline \multicolumn{4}{|l|}{ XVI. Family: CLARIIDAE } \\
\hline \multicolumn{4}{|l|}{ Genus: Clarias Scopoli, 1777} \\
\hline 106. Clarias batrachus (Linnaeus, 1758) & Walking Catfish & Magur & NT \\
\hline 107. Clarias gariepinus (Burchell, 1822)* & African Catfish & African Magur & DD \\
\hline \multicolumn{4}{|l|}{ XVII. Family: HETEROPNEUSTIDAE } \\
\hline Genus: Heteropneutes Muller, 1840 & & & \\
\hline
\end{tabular}

Table 1. Continue 
Table 1. Continued

\begin{tabular}{|c|c|c|c|}
\hline Specific names with Taxonomic position & English name & Local name & $\begin{array}{c}\text { Status of } \\
\text { availability }\end{array}$ \\
\hline $\begin{array}{l}\text { 108. Heteropneustes fossilis (Bloch, 1794) } \\
\text { XVIII. CHACIDAE } \\
\text { Genus: Chaca Gray, } 1831\end{array}$ & Stinging Catfish & Shing, Jiol & NT \\
\hline $\begin{array}{l}\text { 109. Chaca chaca (Hamilton, 1822) } \\
\text { XIX. LORIDAE } \\
\text { Genus: Hypostomus Lacepede, } 1803\end{array}$ & Squarehead Catfish & Chaka & EN \\
\hline $\begin{array}{l}\text { 110. Hypostomus plecostomus (Linnaeus, 1758)* } \\
\text { Order: CYPRINIDONTIFORMES } \\
\text { XX. Family: APLOCHEILIDAE } \\
\text { Genus Aplocheilus McClelland, } 1839\end{array}$ & Plecostomus & Choshok Machh & NL \\
\hline $\begin{array}{l}\text { 111. Aplocheilus panchax (Hamilton, 1822) } \\
\text { Order: SYNBRANCHIFORMES } \\
\text { XXI. Family: SYNBRANCHIDAE } \\
\text { Genus: Monopterus Lacepede, } 1800\end{array}$ & Panchax Minnow & Techoukka & NT \\
\hline $\begin{array}{l}\text { 112. Monopterus cuchia (Hamilton, 1822) } \\
\text { Genus: Ophisternon McClelland, } 1845\end{array}$ & Cuchia & Kuchia & VU \\
\hline $\begin{array}{l}\text { 113. Ophister benglense McClelland, } 1845 \\
\text { Order: PERCIFORMES } \\
\text { XXII. Family AMBASSIDAE }\end{array}$ & Bengal Mud Eel & Kunche & NT \\
\hline $\begin{array}{l}\text { 114. Chanda nama Hamilton, } 1822 \\
\text { Genus: Pseudambassis Bleeker, } 1874\end{array}$ & Glass-perchlet & nama Chanda & VU \\
\hline 115. Pseudambassis baculis (Hamilton, 1822) & Indian Glassy Fish & Kata Chanda & DD \\
\hline 116. Pseudambassis Iala (Hamilton, 1822) & Highfin Glassy Perchlet & Lal chanda & NL \\
\hline $\begin{array}{l}\text { 117. Pseudambassis ranga (Hamilton, 1822) } \\
\text { XXIII. Family: LEIOGNATHIDAE } \\
\text { Genus: Leiognathus Lacepede, } 1803\end{array}$ & Indian Glassy Fish & Ranga Chanda & VU \\
\hline $\begin{array}{l}\text { 118. Leiognathus equulus (Forskal, 1775) } \\
\text { XXIV. Family: SCIAENIDAE } \\
\text { Genus: Otolithoides Fowler, } 1933\end{array}$ & Common Ponyfish & Tak Chanda & NT \\
\hline
\end{tabular}


Bang. J. Anim. Sci. 2009, 38(1\&2)

Table 1. Continued

\begin{tabular}{|c|c|c|c|}
\hline Specific names with Taxonomic position & English name & Local name & $\begin{array}{l}\text { Status of } \\
\text { availability }\end{array}$ \\
\hline $\begin{array}{l}\text { 119. Otolithoides pama (Hamilton,1822) } \\
\text { XXV. Family: NANDIDAE } \\
\text { Genus Nandus Valenciennes, } 1831\end{array}$ & Pama & Poa & NT \\
\hline $\begin{array}{l}\text { 120. Nandus nandus (Hamilton, 1822) } \\
\text { XXVI. Family: PRISTOLEPIDAE } \\
\text { Genus: Badis Bleeker, } 1853\end{array}$ & Mud Perch & Bheda, Meni & VU \\
\hline $\begin{array}{l}\text { 121. Badis badis (Hamilton,1822) } \\
\text { XXVII. Family: CICHLIDAE } \\
\text { Genus: Oreochromis Gunther, } 1889\end{array}$ & Badis & Napit & EN \\
\hline 122. Oreochromis mossambicus (Peters, 1852)* & Tilapia & Tilapia & NL \\
\hline $\begin{array}{l}\text { 123. Oreochromis niloticus (Linnaeus,1758)* } \\
\text { XXVIII. Family: MUGILIDAE } \\
\text { Genus: Rhinomugil Gill, } 1863\end{array}$ & Nile Tilapia & Nilotica & NL \\
\hline $\begin{array}{l}\text { 124. Rhinomugil corsula (Hamilton, 1822) } \\
\text { Genus: Sicamugil Fowler, } 1939\end{array}$ & Corsula Mullet & Khalla, Bata & NT \\
\hline $\begin{array}{l}\text { 125. Sicamugil cascasia (Hamilton,1822) } \\
\text { XXIX. Family: GOBIDAE } \\
\text { Genus: Glossogobius Gill, } 1860\end{array}$ & Yellowtail Mullet & Bata, Keski & NT \\
\hline $\begin{array}{l}\text { 126. Glossogobius giuris (Hamilton, 1822) } \\
\text { Genus: Brachygobius Bleeker, } 1874\end{array}$ & Tank Goby & Bailla & NT \\
\hline $\begin{array}{l}\text { 127. Brachygobius nunus (Hamilton, 1822) } \\
\text { Genus: Gobiopterus Bleeker, } 1874\end{array}$ & Golden-banded Goby & Nuna Baila & NT \\
\hline $\begin{array}{l}\text { 128. Gobiopterus chuno (Hamilton, 1822) } \\
\text { XXX. Family: ANABANTIDAE } \\
\text { Genus: Anabas Cuvier and Cloquet, } 1816\end{array}$ & Glass Goby & Chuno Bele & NT \\
\hline $\begin{array}{l}\text { 129. Anabas testudineus (Bloch, 1775) } \\
\text { XXXI. Family: OSPHRONEMIDAE } \\
\text { Genus Colisa Cuvier, } 1831\end{array}$ & Climbing Perch & Koi & DD \\
\hline $\begin{array}{l}\text { 130. Colisa fasciata (Bloch and Schneider, 1801) } \\
\text { 131. Colisa lalia (Hamilton, 1822) }\end{array}$ & $\begin{array}{l}\text { Giant Gourami } \\
\text { Dwarf Gourami }\end{array}$ & $\begin{array}{c}\text { Khalisha } \\
\text { Lal Khalisha }\end{array}$ & $\begin{array}{l}\text { NT } \\
\text { DD }\end{array}$ \\
\hline
\end{tabular}

Table 1. Continue 
Table 1. Continued

\begin{tabular}{|c|c|c|c|}
\hline Specific names with Taxonomic position & English name & Local name & $\begin{array}{c}\text { Status of } \\
\text { availability }\end{array}$ \\
\hline \multicolumn{4}{|l|}{ Genus Ctenops McClelland, 1845} \\
\hline 132. Ctenops nobilis McClelland, 1845 & Indian Gourami & Neftani & EN \\
\hline \multicolumn{4}{|l|}{ Genus: Trichogaster Schneider, 1801} \\
\hline 133. Trichogaster chuna (Hamilton, 1822) & Honey Gourami & Chuna Khalisha & NT \\
\hline \multicolumn{4}{|l|}{ XXXII. Family: MASTACEMBELIDAE } \\
\hline \multicolumn{4}{|l|}{ Genus: Macrognathus Lacepede, 1800} \\
\hline 134. Macrognathus aculeatus (Bloch, 1786) & Lesser Spiny Eel & Tara Baim & VU \\
\hline 135. Macrognathus pancalus Hamilton, 1822 & Sriped Spiny Eel & Guchi Baim & NT \\
\hline \multicolumn{4}{|l|}{ Genus Mastacebelus Scopoli, 1777} \\
\hline 136. Mastacembelus armatus (Lacepede, 1800) & Tire-track Spiny Eel & Sal Baim & EN \\
\hline \multicolumn{4}{|l|}{ Order: BELONIFORMES } \\
\hline \multicolumn{4}{|l|}{ XXXIII. Family: BELONIDAE } \\
\hline 137. Xenentodon cancila (Hamilton, 1822) & Freshwater Garfish & Kakila & NT \\
\hline \multicolumn{4}{|l|}{ Genus: Hyporhamphus Gill, 1859} \\
\hline 138. Hyporhamphus limbatus (Valenciennes, 1846) & Congaturi Halfbeak & Ek Thuita & NT \\
\hline \multicolumn{4}{|l|}{ Order: TETRODONTIFORMES } \\
\hline \multicolumn{4}{|l|}{ XXXIX. Family: TETRADONTIDAE } \\
\hline \multicolumn{4}{|l|}{ Genus: Tetraodon Linnaeus, 1758} \\
\hline 139. Tetraodon cutcutia Hamilton, 1822 & Common Pufferfish & Tepa, Potka & NT \\
\hline
\end{tabular}

Status of availability: EN- Endangered, VU- Vulnerable, CR- Critically endangered

DD- Data deficient, NT- Not threatened, NL- Not listed

* Exotic species introduced in freshwater of Bangladesh

As mentioned earlier Bhuiyan (1964) gave an account of 71 species of freshwater fishes belonging 45 genera and 25 families which he collected from Dhaka. Doha (1973) published a check-list (probably incomplete) of 106 species of fishes collected from the districts of Mymensingh and Tangail belonging to 68 genera and 34 families including a single exotic species. Chandra and Aminul Haque (1982a, 1982b) confirmed the availability of two species of Mahashol Tor tor and Tor putitura in Bangladesh waters. Haque (2004) described 134 species of fishes under 76 genera and 32 families from the capture fisheries of the Padma river. Rahman (2005) compiled a list of 265 species of freshwater fishes belonging to 154 genera and 55 families. Siddiqui et al. (2007) described 251 species of freshwater fishes belonging to 61 families from Bangladesh. Considering the number of families, genera and species of fishes, it appears from the present investigation that districts of greater Mymensingh are still rich in freshwater fish 
Bang. J. Anim. Sci. 2009, 38(1\&2)

fauna. Thirteen exotic species available are also included in the present compilation. According to IUCN (2000), among the 126 indigenous fish species of Mymensingh, 26 are endangered, 11 are vulnerable and 10 are critically endangered. The other 19 species with data deficient, 5 species are not listed, and the rest 55 species are not threatened. The critically endangered species are Nandil, Bhangan, Sarpunti, Mahashol, Rita, Ghaura, Bacha, Pangas, Baghair and Tilshol.

\section{Conclusion}

The present work represents the fish fauna found to inhabit the water bodies of greater Mymensingh. In view of number of species, genera and families of fishes reported herein it seems that Mymensingh region is still rich in freshwater fish fauna though many indigenous species are dwindling day by day. Further works are, of course, needed on the systematics, ecology, distribution, habits and habitats of these fish fauna of greater Mymensingh region.

\section{Literature Cited}

Ahmed, M. A. and Hasan, S. 1981. A Check-list of the fishes of the Karnaphuli reservoir. Bangladesh J. Zool., 9(1): 37-40.

Akter, S. 2009. Landing, Marketing System and Fisheries Fauna in Mymensingh Town.

M. S. Thesis, Department of Aquaculture, Bangladesh Agricultural University, Mymensingh. $56 \mathrm{pp}$.

Alam, S. 2008. Assessment Livelihood status of Fishers of Bamunji beel in Jamalpur district. M. S. Thesis, Department of Fisheries Management, Bangladesh Agricultural University, Mymensingh. 88 pp.

Bhuiyan, A. L 1964. Fishes of Dacca. Asiatic Society of Pakistan, Dacca. 148 pp.

Chakaborty, B. K. and Mirza, J. A. 2006. Study of Aquatic Biodiversity of Nethai river in Bangladesh. J. Crop. Weed. 2(2): 20-30.

Chandra, K. J. and A. K. M. Aminul Haque. 1982a. Studies on the biology of Tor spp. in natural and artificial waters of Bangladesh. i. Distribution, Abundance, Fishery. Bangladesh J. Fish., 2-5 (1-2): 51-58.

Chandra, K. J. and A. K. M. Aminul Haque.1982b. Studies on the biology of Tor spp. in natural and artificial waters of Bangladesh. ii. Taxonomy. Bangladesh J. Fish., 2-5 (59-64).

Debnath, P. C. 2009. A Study on the Fish Biodiversity of Borulia Haor, Nikli, Kishoreganj and the Socioeconomic condition of the Adjacent Fishers. M. S. Thesis, Department of Aquaculture, Bangladesh Agricultural University, Mymensingh. 49 pp.

Doha, S. 1973. Fishes of the districts of Mymensingh and Tangail. Bangladesh J. Zool. 1: 1-10.

Dowla, M. A. U. 2008. Socioeconomic condition of Fishermen of the Jamuna River in Dewanganj Upazilla under Jamalpur district. M. S. Thesis, Department of Fisheries Management, Bangladesh Agricultural University, Mymensingh. 63 pp.

Hamilton, F. 1822. An Account of the Fishes Found in the River Ganges and its Branches. Archibald Constable and Company, Edinburgh. 405. pp. 
Hossain, M. S., Das, N. G. and Chowdhury, M. S. N. (eds.). 2007. Fisheries Management of the Naaf River. Coastal and Ocean Research Group of Bangladesh (CORG), Inst. Mar. Sci. Fish., University of Chittagong, Chittagong, Bangladesh. 257 pp.

Faroque, M. G. 2006. Fisheries in Borobela beel in Mymensingh district and Livelihood of the adjacent Fisheries and Fish Farmers. M. S. Thesis, Department of Fisheries Management, Bangladesh Agricultural University, Mymensingh. 34 pp.

Haque, M. A. 2004. Capture Fisheries of the river Padma near Rajshahi. Ph.D. Thesis, Institute of Biological Sciences. Rajshahi University, Bangladesh. 180 pp.

Hossain, T. 2007. Study on Biodiversity of Fish fauna and Socioeconomic Condition of the Fishermen in the Kolimar Haor, Itna, Kishoreganj. M.S. Thesis, Department of Fisheries Management, Bangladesh Agricultural University, Mymensingh. 38 pp.

Islam, M. S. and Hossain, M. A. 1983. An account of the fishes of the Padma near Rajshahi. Fish. Bull. 1(2): 1-31.

IUCN Bangladesh. 2000. Red Book of Threatened Fishes of Bangladesh. IUCN-The World Consevation Union. $116 \mathrm{pp}$.

Rahman, A. K. A. 1989. Freshwater Fishes of Bangladesh. Zoological Society of Bangladesh, Dhaka. 364 pp.

Rahman, A. K. A. 2005. Freshwater Fishes of Bangladesh. 2nd ed. Zoological Society of Bangladesh, Dhaka. $394 \mathrm{pp}$.

Rahman, M. M. 2000. Comparison of Benthic Fauna of two beels of Netrakona district under Different Management condition. M. S. Thesis, Department of Aquaculture, Bangladesh Agricultural University, Mymensingh. $75 \mathrm{pp}$.

Rahman, M. M. 2009. Fishing Operations and Biodiversity of Fish Fauna of Katar Beel in Fulbaria Upazilla of Mymensingh district. M. S. Thesis Department of Aquaculture, Bangladesh Agricultural University, Mymensingh. 40 pp.

Shahjahan, M., Miah, M. I. and Haque, M. M. 2001. Present status of Fisheries in the Jamuna River. Pak. J. Biol. Sci. 4(9): 1173-1176.

Siddiqui, K. U., Islam, M. A., Kabir, S. M. H., Ahmad, M., Ahmed, A. T. A., Rahman, A. K. A., Haque, E. U., Ahmed, Z. U., Begum, Z. N. T., Hasan, M A., Khondker, M. and Rahman, M. M. (eds.). 2007. Encyclopedia of Flora and Fauna of Bangladesh. Vol. 23. Freshwater Fishes. Asiatic Society of Bangladesh, Dhaka 300 pp.

Talwar, P. K. and Jhingran, A. G. 1991. Inland Fishes of India and Adjacent Countries. Vol. 1 \& 2. Oxford \& IBH Publishing Co. Pvt. Ltd., New Delhi, India. 1158.

Wahab, M. A. 2003. Small indigenous fish species of Bangladesh. Potentials for culture and conservation. In: Wahab, M. A., Thilsted, S. H. and Hoq, M. E. (eds.), Small Indigenous Species of Fish in Bangladesh: Culture Potentials for Improved Nutrition \& Livelihood. Bangladesh Agricultural University, Mymensingh, Bangladesh. pp. 1-12. 